\title{
Acute pancreatitis due to ramipril therapy
}

M Kanbay, M Korkmaz, U Yılmaz, G Gur, S Boyacioglu

Postgrad Med J 2004;80:617-618. doi: 10.1136/pgmj.2003.018119

Few data exist about the incidence of drug induced acute pancreatitis in the general population. Drugs are related to the aetiology of pancreatitis in about $1.4 \%-2 \%$ of cases. Although angiotensin converting enzymes are generally well tolerated, acute pancreatitis has been reported in a few subjects treated with captopril, enalapril, and lisinopril. A 85 year old man with a long standing history of hypertension, who was treated with ramipril $5 \mathrm{mg}$ once daily, presented with acute pancreatitis. Other causes of the disease were ruled out. After cessation of ramipril his condition improved and amylase level decreased. This was his third episode of acute pancreatitis since ramipril was started in 2000 . To the authors' knowledge ramipril induced pancreatitis has not previously been reported.

$\mathrm{D}$ rugs are related to the aetiology of pancreatitis in around $1.4 \%-2 \%$ of cases. Angiotensin coverting enzyme (ACE) inhibitors are generally well tolerated, but acute pancreatitis has been reported in a few cases treated with lisinopril, captopril, and enalapril. To our knowledge, ramipril induced pancreatitis has not previously been reported. We report a case of recurrent pancreatitis, which was a severe and life threatening allergic reaction to ramipril. Because this drug may come into more widespread clinical use, we wish to alert clinicians and urge close monitoring for pancreatitis as well as other adverse effects.

\section{CASE REPORT}

A 85 year old man was monitored for hypertension and had been treated with ramipril ( $5 \mathrm{mg}$ ) since 2000 . His first acute episode of pancreatitis was eight months after starting ramipril and the second was 11 months after the first acute pancreatitis attack. The episode of acute pancreatitis reported here was the third within 26 months of starting the drug. Previous investigations had included abdominal ultrasonography and endoscopic retrograde cholangiopancreatography and common causes of pancreatitis had been discounted.

He presented on this occasion with severe epigastric pain radiating to his back for the previous two days. The pain was accompanied by nausea and vomiting, which was white, intermittently green with no blood. The patient reported no diarrhoea, gas, or fever. He denied alcohol use and smoking. On physical examination, the abdomen was distended and tenderness was present in the epigastrium. There was no rebound tenderness, rigidity, or guarding. Laboratory test results revealed increased serum levels of amylase: $1261 \mathrm{U} / \mathrm{l}$ (reference range 25-115), lipase: $1368 \mathrm{U} / \mathrm{l}$ (40-110), and Creactive protein: $84 \mathrm{mg} / \mathrm{l}(0-6)$. Serum values of liver enzymes and leucocytes were normal. Abdominal ultrasound showed that the head of the pancreas was oedematous and hypoechoic. The biliary tree was not dilated and no gallstones were seen. He was diagnosed with acute pancreatitis. Possible common causes of acute pancreatitis such as heavy alcohol intake, hypercalcaemia, hypertriglyceridaemia, neoplasia, viral infections, and abdominal trauma were also excluded. Ramipril was stopped and he received symptomatic medical treatment. The patient's clinical state spontaneously improved in 48 hours. His serum amylase level returned to normal on day 4 after cessation of ramipril and he was discharged from hospital six days later. Ramipril was replaced with atenolol; he was well when seen at follow up. He has not had a new pancreatitis attack for 14 months.

\section{DISCUSSION}

In this report we describe a patient who had no risk factors for pancreatitis and was taking no medications known to cause pancreatitis other than ramipril. Drug induced pancreatitis has no distinguishing features. The occurrence of reported cases of pancreatitis secondary to ACE inhibitors is relatively rare and mostly associated with captopril, lisinopril, and enalapril. ${ }^{1-3}$ ACE inhibitors are first line agents in cardiovascular disease. The pathogenesis of drug induced pancreatitis may be caused by an allergic response or by a direct toxic effect. ACE inhibitors work against high blood pressure because they inhibit an enzyme that is necessary to produce a substance that pushes the blood pressure up. But this same action seems to inhibit the breakdown by enzymes of substances in the body that can make blood vessels leaky. Therefore, secretions of the pancreas may cause acute pancreatitis. Angioedema is at best an uncomfortable and disfiguring type of temporary swelling. Pancreatitis associated with ACE inhibitors is thought to reflect localised angioedema of the gland. ${ }^{1-3}$ Angioedema due to ACE inhibitors appears to be linked to the decreased degradation of bradykinin because ACE, also known as kininase II, not only activates angiotensin I but also inactivates bradykinin. Angiotensin II receptors are thought to be important in the regulation of pancreatic secretion and microcirculation, and this may have an additive effect on pathogenesis. ${ }^{4}$ The time course of developing the disorder varies from a few weeks to years after starting the drug. Proving the association with a particular drug may not always be straightforward, even in suspected cases. Thus, patients restarted on their medications should be closely monitored and the drug promptly discontinued if symptoms recur. ${ }^{5}$ Reintroduction of ramipril therapy resulted in the recurrence of pancreatitis, and this strongly supports the association. Moreover, there were no further attacks in our patient after 14 months of follow up with no intake of ramipril. Early recognition of this reaction is crucially important both for rapid discontinuation of the offending drug and for the avoidance of unnecessary drug therapy or invasive procedures.

In conclusion, to the best of our knowledge, this is the first report in the literature of ramipril induced pancreatitis. With increased use of this drug further reports may shed light on the incidence, clinical importance, and mechanism of this adverse drug reaction. In addition to monitoring for efficacy and commonly reported adverse effects, clinicians need to be aware that acute pancreatitis may occur in patients taking ACE inhibitors. 


\section{Authors' affiliations}

M Kanbay, Department of Internal Medicine, Baskent University Faculty of Medicine, Ankara, Turkey

M Korkmaz, U Yılmaz, G Gur, S Boyacioglu, Department of Gastroenterology, Baskent University Faculty of Medicine, Ankara, Turkey

Correspondence to: Dr Mehmet Kanbay, 35 Sokak, 81/5, Bahcelievler 06490, Ankara, Turkey; drkanbay@yahoo.com

Submitted 14 December 2003

Accepted 28 April 2004

\section{REFERENCES}

1 Standridge JB. Fulminant pancreatitis associated with lisinopril therapy. South Med J 1994;87:179-81.

2 Maliekal J, Drake CF. Acute pancreatitis associated with the use of lisinopril. Ann Pharmacother 1993;27:1465-6.

3 Gershon T, Olshaker JS. Acute pancreatitis following lisinopril rechallenge. Am J Emerg Med 1998; 16:523-4.

4 Fisher AA, Bassett ML. Acute pancreatitis associated with angiotensin II receptor antagonists. Ann Pharmacother 2002;36: 1883-6.

5 Runzi M, Layer P. Drug-associated pancreatitis: facts and fiction. Pancreas 1996;13:100-9. 